\title{
Erratum to: Bone Matrix Maturation in a Rat Model of Intra-Cortical Bone Remodeling
}

\author{
Ryan D. Ross $^{1} \cdot$ D. Rick Sumner ${ }^{1,2}$
}

Published online: 29 April 2017

(C) Springer Science+Business Media New York 2017

\section{Erratum to: Calcif Tissue Int}

DOI 10.1007/s00223-017-0270-7

The captions for Figs. 5 and 6 were interchanged in the original publication. The correct versions of Figs. 5 and 6 are published with this erratum.

The original article has been corrected.

The online version of the original article can be found under doi:10.1007/s00223-017-0270-7.

$\triangle$ D. Rick Sumner

Rick_Sumner@rush.edu

1 Department of Cell \& Molecular Medicine, Rush University Medical Center, Chicago, IL 60612, USA

2 Department of Orthopedic Surgery, Rush University Medical Center, Chicago, IL, USA 


\section{Virgin/Normal Ca}
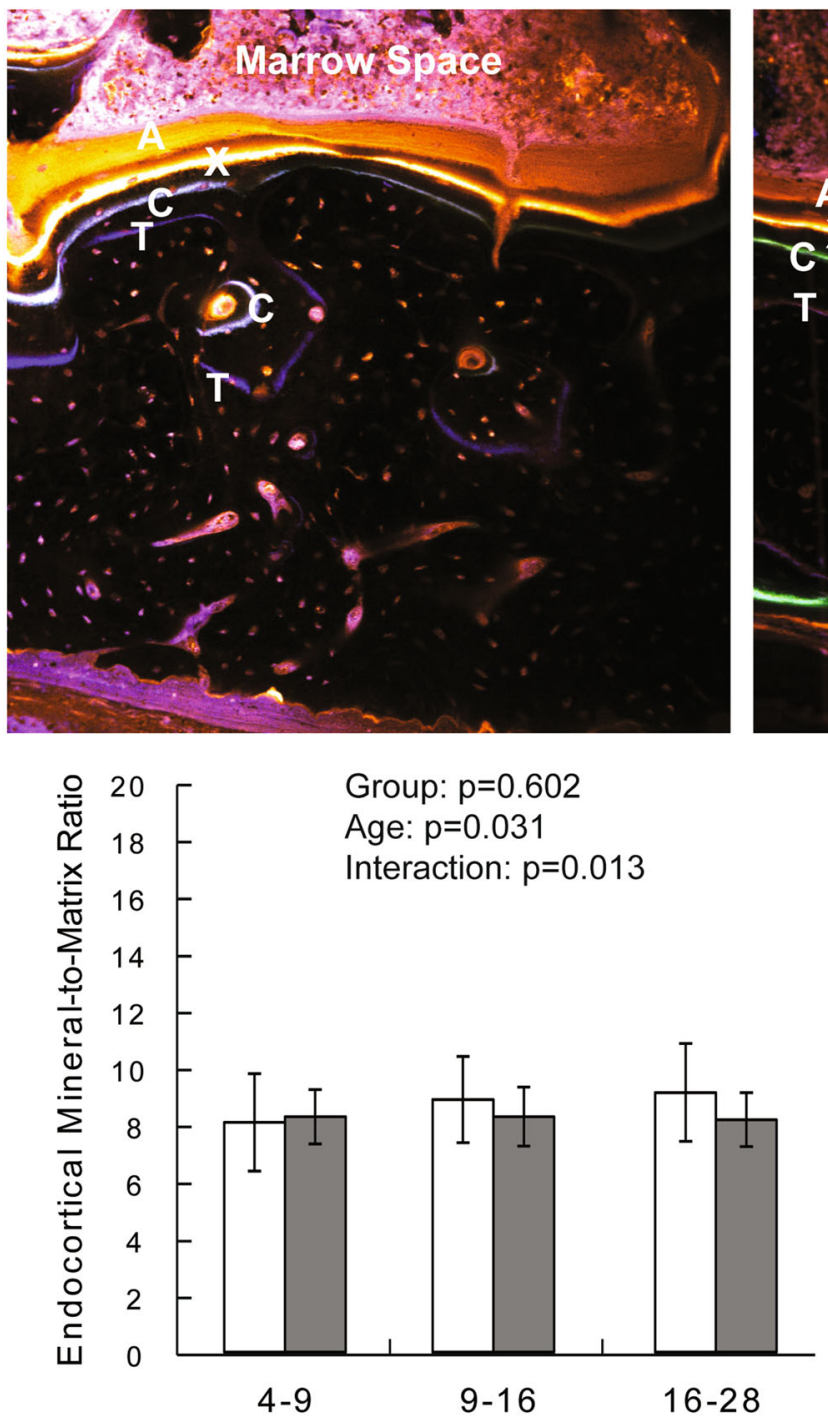

Tissue-age (days)

\section{Lactation/Low Ca}
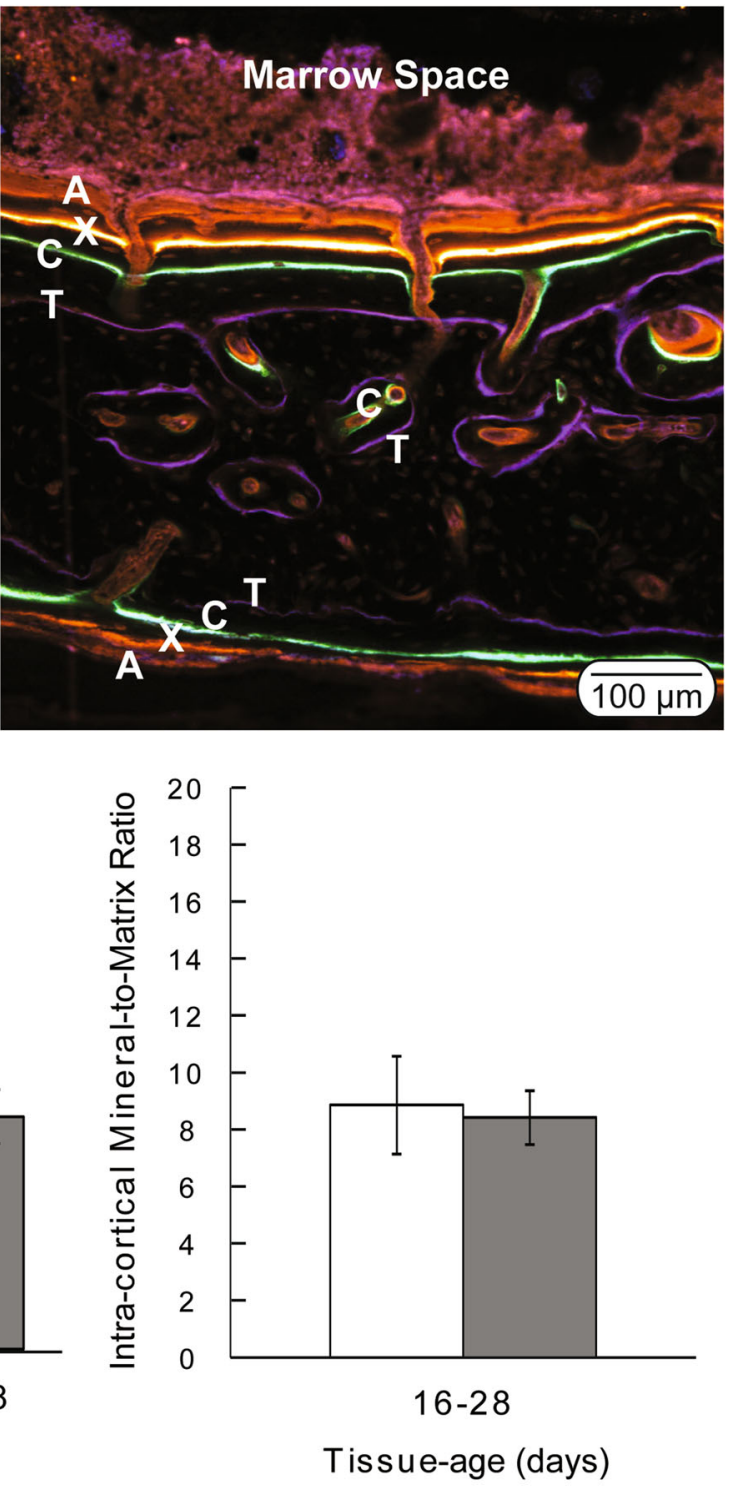

Lactation/Low Ca
Fig. 5 (Top) Representative confocal microscopy images demonstrating the presence of fluorochrome labels in the endocortical and intra-cortical compartments of both virgin/normal $\mathrm{Ca}$ and lactation/ low-Ca groups. The virgin/normal Ca controls lacked fluorochrome labelsin the periosteal compartment. Oxytetracycline (labeled ' $\mathrm{T}$ ' in the images) was given on day 2 ; calcein ('C') was given on day 14 ; xylenol orange (' $\mathrm{X}$ ') was given on day 21 ; and alizarin ('A') was given on day 26 of the recovery phase. (Bottom) Degree of mineralization (mineral-to-matrix ratio) plotted as a function of tissue age and compartment. Data are presented as the means and standard deviations. There was not a significant difference between groups in the intra-cortical compartment. Endocortically, the two-way ANOVA indicated a significant tissue age effect $(p=0.031)$ and a significant interaction term $(p=0.013)$, with no significant group effect $(p=$ 0.602 ) 

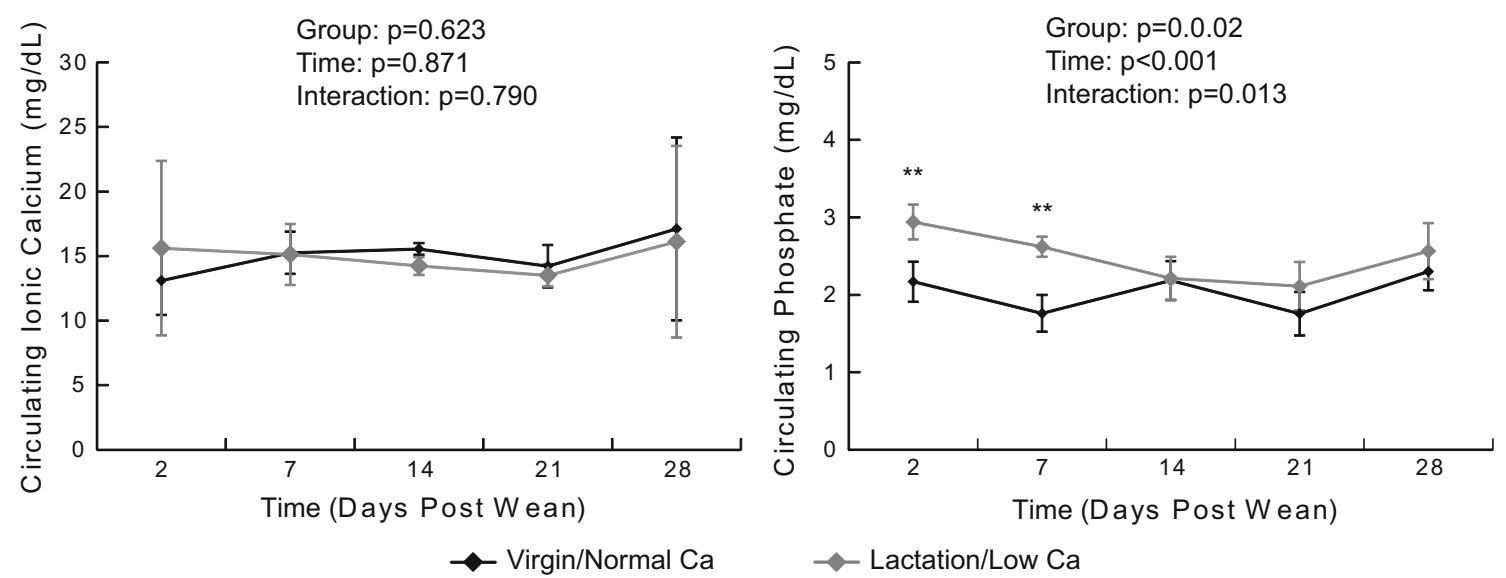

Fig. 6 Circulating calcium and phosphate concentration measured longitudinally during the recovery phase (means and standard deviations, $n=$ 4-5 per group per time point). Results from the repeated measures ANOVA are presented in the legend 